\title{
Rhabdomyosarcoma of thumb: A case report with review of literature
}

\author{
S. Raja Sabapathy, Hari Venkatramani, S. Udhaya Shankar, Sanjai Ramkumar \\ Department of Plastic, Hand and Reconstructive Microsurgery, Ganga Hospital, 313, Mettupalayam Road, Coimbatore, \\ Tamil Nadu, India
}

Address for correspondence: Dr. S Raja Sabapathy Department of Plastic, Hand and Reconstructive Microsurgery, Ganga Hospital, 313, Mettupalayam Road, Coimbatore -641 043, Tamil Nadu, India. E-mail: rajahand@vsnl.com

\section{ABSTRACT}

Rhabdomyosarcoma is a rare malignant tumour occurring in the hand. Though the prognosis for this tumour is poor, when it occurs in the hand, early diagnosis is possible, since the swelling becomes clinically obvious. Radical surgical excision even at the cost of significant functional loss is recommended with possible reconstruction once the disease is under control. Advances in chemotherapy protocols and radiotherapy in selected cases has improved the prognosis.

Rhabdomyosarcoma arising in the thumb of a four-year-old child is presented. He was treated by amputation of the thumb at the carpometacarpal joint level followed by chemotherapy. When he was disease-free at 18 months, thumb was reconstructed by pollicisation of the index finger. The child has remained recurrence-free at 30 months from diagnosis. The patient details are presented with review of the relevant literature.

\section{KEY WORDS}

Hand tumour, pollicisation, rhabdomyosarcoma

habdomyosarcoma is a rare, highly malignant tumour occurring in the paediatric age group. It accounts for nearly $65 \%$ of all sarcomas in patients of 15 years of age and younger and approximately $7 \%$ of all childhood malignant solid tumours. ${ }^{[1]}$ About $20 \%$ of the reported cases involve the extremities. It was an uncommonly entertained diagnosis in the hand until the publication of Potenza and Winslow in 1961. ${ }^{[2]}$ They presented two of their cases and added seven unpublished cases sourced from the records of the US Armed Forces Institute of Pathology. Earlier to their publication there were only three documented cases of rhabdomyosarcoma arising from the skeletal muscles of the hand.

These tumours have been classified into four subtypes on a histological basis: embryonal, alveolar, botryoid and pleomorphic. ${ }^{[3]}$ Embryonal types in children which frequently affect the bladder and head and neck have better prognosis. Alveolar type is the most common type that involves the extremities and has got poorer prognosis. Intergroup Rhabdomyosarcoma Study Group (IRSG) has evolved a staging system for this childhood tumour ${ }^{[4]}$ [Tables 1 and 2]. It incorporates the elements of Tumour, Node and Metastases, but also lays emphasis on the site of occurrence of the primary tumour. This is because the site plays a major determinant in the recurrence and the prognosis of the disease. Since most of the extremity rhabdomyosarcoma belong to the alveolar type in histology, it is associated with poor prognosis and hence at the time of clinical diagnosis most of them belong to Stage II. Survival statistics 
Table 1: TNM pretreatment staging system (intergroup rhabdomyosarcoma study group)

\begin{tabular}{|c|c|c|c|c|c|}
\hline Stage & Sites & $T$ & Tumour size & $N$ & $M$ \\
\hline \multirow[t]{3}{*}{ I } & Orbit & $\mathrm{T} 1 \mathrm{or} \mathrm{T} 2$ & $a$ or b & $\mathrm{N} 0$ or N1 or N2 & MO \\
\hline & Head and neck (excluding parameningeal) & & & & \\
\hline & GU-Nonbladder/Nonprostate & & & & \\
\hline \multirow[t]{4}{*}{ II } & Bladder/Prostate & T1 or T2 & a & $\mathrm{N} 0$ or $\mathrm{Nx}$ & M0 \\
\hline & Extremity & & & & \\
\hline & Head and neck parameningeal & & & & \\
\hline & Other (including trunk, retroperitoneum, etc.) & & & & \\
\hline \multirow[t]{4}{*}{ III } & Bladder/Prostate & T1 or T2 & a & N1 & M0 \\
\hline & Extremity & & & & \\
\hline & Head and neck parameningeal & & b & $\mathrm{N} 0$ or $\mathrm{N} 1$ or $\mathrm{Nx}$ & \\
\hline & Other (including trunk, retroperitoneum, etc.) & & & & \\
\hline IV & All & T1 or T2 & $a$ or $b$ & N0 or N1 & M1 \\
\hline
\end{tabular}

Table 2: Definitions of $\mathrm{T}, \mathrm{N}$ and $\mathrm{M}$ classifications in pretreatment staging

\begin{tabular}{ll}
$\begin{array}{ll}\text { Classification } \\
\text { Tumour }\end{array}$ & Description \\
T1 & Confined to anatomic site of origin \\
a & $<5 \mathrm{~cm}$ in diameter \\
b & $\geq 5 \mathrm{~cm}$ in diameter \\
T2 & Extension and/or fixation to surrounding \\
& tissue \\
a & $<5 \mathrm{~cm}$ in diameter \\
b & $\geq 5 \mathrm{~cm}$ in diameter \\
Regional lymph nodes & \\
N0 & Regional lymph nodes not clinically involved \\
N1 & Regional lymph nodes clinically involved \\
& by neoplasm \\
Nx & Clinical status of regional lymph nodes \\
& unknown (especially with sites that preclude \\
Metastasis & lymph node evaluation) \\
M0 & No distant metastasis \\
M1 & Metastasis present \\
\hline
\end{tabular}

Table 3: Survival of IRS-II and IRS-III Patients by intergroup rhabdomyosarcoma study group pretreatment stage

\begin{tabular}{lcc}
\hline Pretreatment stage & \multicolumn{2}{c}{ Estimated 5-year survival } \\
\cline { 2 - 3 } & IRS-II (\%) & IRS-III (\%) \\
I & 91 & 89 \\
II & 73 & 86 \\
III & 52 & 69 \\
IV & 23 & 30 \\
\hline
\end{tabular}

according to the pre-treatment stage in the IRS group are given in Table 3.

Though these tumours apparently carry a poor prognosis, when it occurs in the hand it is quite possible to have a long-term disease-free state by a combination of early detection and appropriate treatment. This point is particularly important in tumours of the hand since the swelling becomes clinically obvious early in the course of the disease.
A case of alveolar rhabdomyosarcoma in the thumb in a four-year-old child treated by amputation followed by chemotherapy and subsequent reconstruction with pollicisation is presented. The child is well with a 30 month disease-free interval state. The relevant literature is reviewed.

\section{CASE REPORT}

A four-year-old right handed boy was seen by his family physician with pain and swelling of right thumb following a fall. Radiograph revealed fracture of proximal phalanx for which a plaster of Paris slab was applied. Three weeks later when the slab was removed the swelling was still present with unhealed fracture and hence was referred to an Orthopaedic Surgeon. A soft tissue swelling $2 \mathrm{~cm} \times 1 \mathrm{~cm}$ at the level of the metacarpo phalangeal joint was present with a lytic lesion of the proximal phalanx in the X-ray. A biopsy was performed and a diagnosis of alveolar rhabdomyosarcoma was confirmed. Immunohistochemistry revealed that the tumour cells were strongly positive for Myoglobin [Figure 1]. There was focal positivity for NSE (Neuron-Specific-Enolase), S-100 and tumour was negative for Cytokeratin and Leucocyte Common Antigen (LCA). Ultrasonogram of abdomen and chest, whole body bone scan were performed and there was no other area of involvement. A CT scan of the thumb showed osteolytic lesion in the base of the phalanx of the thumb with minimal soft tissue swelling on the lateral aspect of the thumb suggestive of a neoplastic lesion. At this stage the boy was referred to our centre [Figure 2].

On consultation with the oncologist it was decided that the best prognosis would be possible with radical surgical clearance. Though it would involve amputation of the thumb we recommended it since the disease 


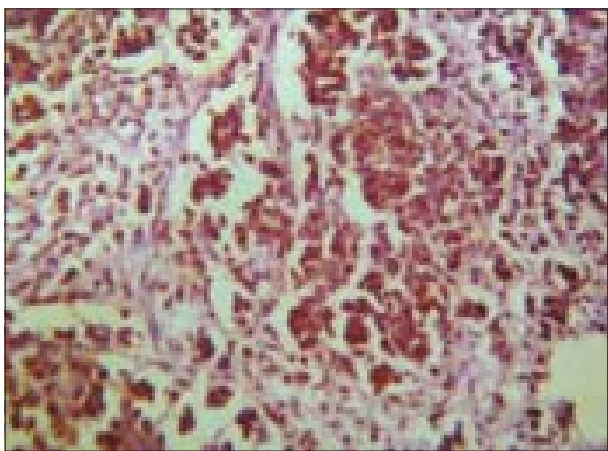

Figure 1: Tumour cells showing strong positivity for Myoglobin

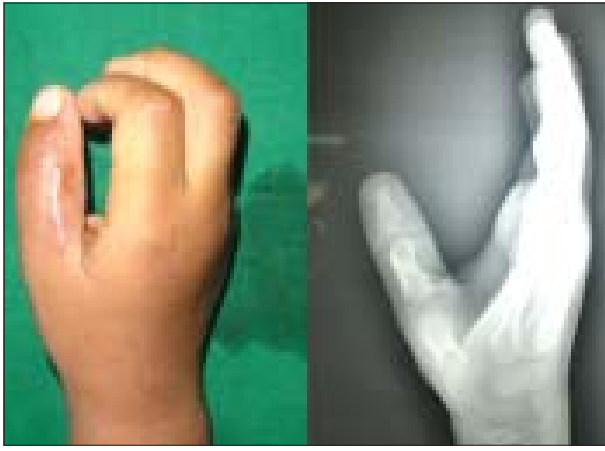

Figure 2: The thumb showing healed biopsy scar and the radiograph with irregularity of the base of the proximal phalanx was restricted to the hand with no obvious metastasis. Amputation of thumb was performed at the CarpoMetacarpal joint [Figures 3a-c]. It was followed up by four cycles of chemotherapy with vincristine and actinomycin spread over a period of one year.

Eighteen months after surgery a full clinical, radiological and imaging workup was done. No local recurrence or metastasis was detected. Absence of thumb was causing considerable disability, since it involved the right dominant hand and the parents wanted to put him in school. Pollicisation of the index finger was done and the patient went through regular physical therapy. On follow-up, at 30 months after diagnosis, the child remains disease-free and using his pollicised thumb fully for all activities [Figures 4a-c].

\section{DISCUSSION}

In a review of 200 cases of sarcomas Hark and Cerney found that only $5 \%$ of them were rhabdomyosarcomas. ${ }^{[5]}$ Occurrence of skeletal muscle tumours as compared to tumours arising from other tissues is quite low when the relative amount of skeletal muscle per pound of human
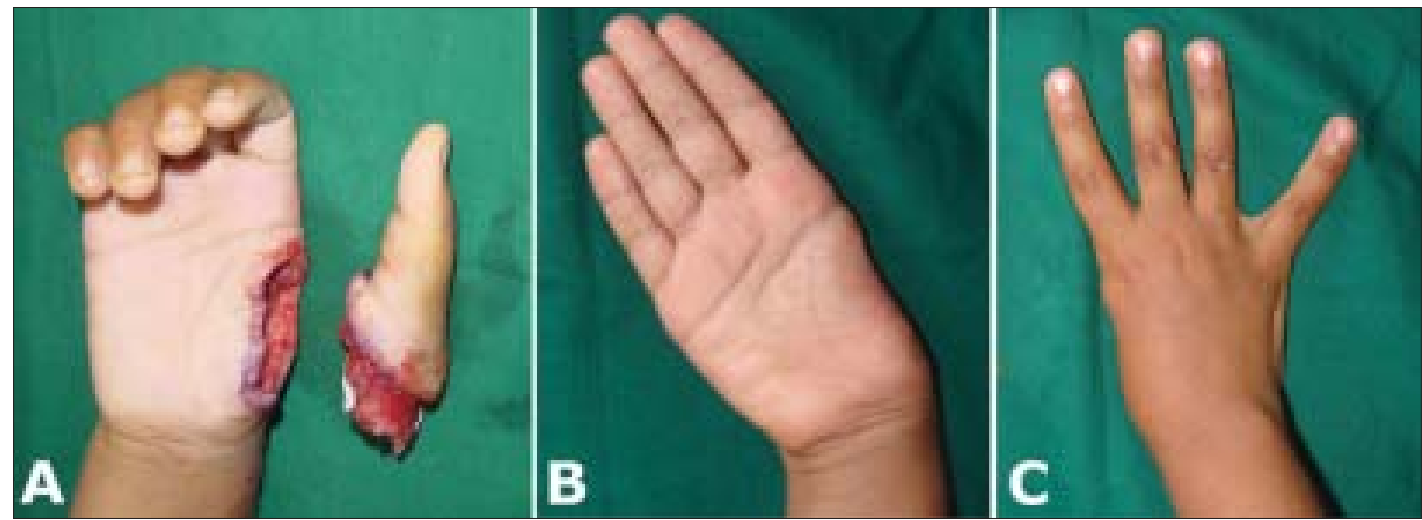

Figure 3 A-C: A- The amputation of the thumb at the CMC Joint. B \& C. - The view of the hands before pollicisation
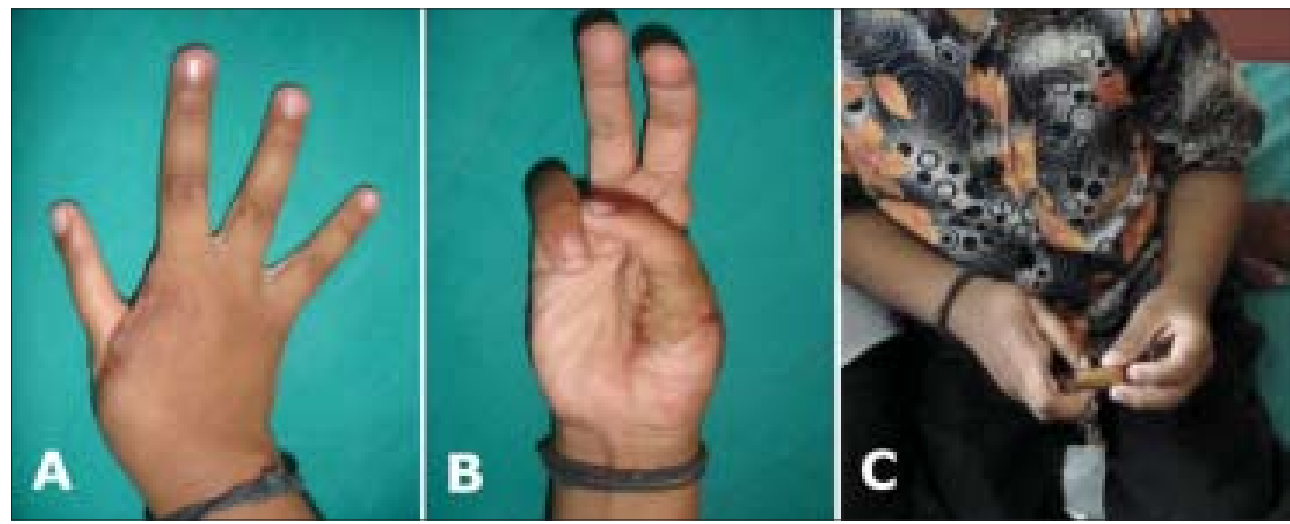

Figure 4 A-C: The pollicised thumb at one-year follow-up and the child is using it for all activities 
tissue is concerned. This is apparently due to the fact that the skeletal muscle cells are completely differentiated and do not undergo cellular division in postnatal life as do those of other tissues. ${ }^{[5]}$

Rhabdomyosarcoma in the hand is even rarer and the first major report was that of Potenza and Winslow. All their cases with good follow-up were uniformly fatal and none of them had chemotherapy. Linscheid did a clinico-pathological study of the rhabdomyosarcomata of the extremities and limb girdles, seen at Mayo clinic, between 1905-1961. ${ }^{[6]}$ A total of 87 cases of pleomorphic rhabdomyosarcoma of the extremities were encountered of which five tumours were located in the hand. They emphasized radical excision as an important component of treatment. The appearance of encapsulation might tempt surgeons to try local excision, but the folly of this approach has been reemphasized in many studies. In Linscheid's study 59 patients had a total of 134 recurrences which serves to emphasize the unsuitability of local excision for this neoplasm. Radical operation offers the best chance of survival, which is attested by the fact that of 30 survivors in their series, 25 had had either amputation or radical en bloc excision. They also concluded that lesions of the upper extremity had better prognosis than the lesions of the lower extremity and more distal lesions had a better prognosis than the more proximal lesions.

Xarchas et al. reported two cases with tumours arising in the ulnar border of the hand. ${ }^{[7]}$ One patient had local excision followed by chemotherapy, but succumbed to the disease 16 months after surgery. The other 29year-old patient had ulnar hemi-amputation followed by four intensive courses of combination chemotherapy using Vincristine, Adriamycin and Cyclophosphamide (VAC) along with radiotherapy. When the patient was progressing well without any recurrence, at 14 months tendon transfers were performed to correct significant radial deviation of the carpus. At 4.5 years follow-up there was no evidence of local or general recurrence. The patient subsequently got married and delivered a healthy child. They emphasize the point that patients with tumours in the extremities benefit from total excision of the tumour even at the cost of significant functional loss and cosmetic blemish. With the available reconstructive techniques functional reconstruction at alater stage can be performed. Our case also emphasizes the same point wherein we obtained radical surgical clearance with amputation of the thumb at the CMC joint level and later reconstructed the thumb with pollicization. Chemotherapy forms an important component of treatment. Most authors do not consider radiotherapy to be of great use once radical surgical ablation or amputation is done.

While the average age of children affected is around eight years, this has also been documented to present at birth. Cohen ${ }^{[8]}$ reported a child with Apert's syndrome with congenital embryonal rhabdomyosarcoma in the hand. At birth the child had a $1 \mathrm{~cm}$ soft bluish mass in the thenar eminence of the right palm which was thought of as a haemangioma. At 14 months it enlarged and intralesional steroids were injected but the tumour continued to grow. Soon a mass in the forearm and a node in the axilla appeared and biopsy revealed the diagnosis. All the masses were radically excised and the child was followed with chemotherapy and radiotherapy. At publication time of 18 months the child was disease-free.

Synchro nousappearanceofrhabdomyosarcomasinmultiple sites has also been reported. Siegel et al. ${ }^{[9]}$ reported an 11year-old child presenting with rhabdomyosarcomas in the hand and the thigh. This child had biopsy of the tumours followed by neoadjuvant chemotherapy, which consisted of 12 weeks of treatment with vincristine, actinomycin and cyclophosphamide. The MR scan performed after this showed that the thigh mass had disappeared although the hand mass was only slightly smaller. The hand mass and thigh tumour bed were widely resected. The hand resection consisted of hemi-metacarpal resection of the middle and index finger with autogenous tricortical iliac crest grafting. Postoperatively the patient received external beam radiation as well as chemotherapy. At $30-$ month follow-up the patient had near normal function of the hand with no evidence of disease. They recommend chromosomal studies at the time of biopsy to predict the response to chemotherapy.

Response to chemotherapy may be predicted by the identification of gene fusions and chromosomal rearrangement. In cases of rhabdomyosarcoma the presence of one or the other of the gene fusions has been shown to have prognostic significance, because they can distinguish between a very high risk subgroup (PAX3-FKHR) and a favourable outcome subgroup (PAX7FKHR). Multivariate analysis demonstrated a significantly increased risk of failure $(P=0.025)$ and death $(P=0.019)$ in patients with metastatic disease if their tumours expressed PAX3-FKHR. ${ }^{[10]}$

Many of the extremity tumours in the literature had 
metastasis at the time of initial presentation. They had a poor prognosis irrespective of the treatment modality. The subgroup of rhabdomyosarcoma primarily occurring in the hand could have a good prognosis because they can be detected early. ${ }^{[11]}$ Surgical technique plays an important role. The incision used for the biopsy must be longitudinal and must be the one which will be excised during definitive surgery.

Exsanguination with tourniquet is not recommended for fear of causing seeding of the tumour. We use tourniquet with elevation of the hand before inflation and this has not been scientifically proven to cause any problem.

For the tumours of the hand radical excision holds the best prospect of cure. This is preferred even at the cost of functional disability. Reconstructive surgery could be done later to enhance function. Radiotherapy after the amputation of the primary area has not found to be of significant use, but could be given when local wide excision is done. All cases must have chemotherapy. The overall survival from this tumour is improving currently because of the use of therapeutic agents such as vincristine, actinomycin-D, cyclophosphamide and adriamycin plus cisplatinum, as well as external beam radiation therapy and high-dose methotrexate. For tumours locally controlled with surgical resection, fiveyear survival approaching $70 \%$ has been reported. ${ }^{12]}$ The current advances in chromosomal analysis can predict the favourable and unfavourable subtypes of rhabdomyosarcoma.

\section{REFERENCES}

1. Young JL Jr, Ries LG, Silverberg E, Horm JW, Miller RW. Cancer incidence, survival and mortality for children younger than age 15 years. Cancer 1986;58:598-602.

2. Potenza AD, Winslow DJ. Rhabdomyosarcoma of the hand. J Bone Joint Surg Am 1961;43:700-8.

3. Murray PM. Soft tissue sarcoma of the upper extremity. Hand Clin 2004;20:325-33.

4. Lawrence W Jr, Anderson JR, Gehan EA, Maurer H. Pretreatment TNM staging of childhood rhabdomyosarcoma: A report of intergroup rhabdomyosarcoma study group. Cancer 1997;80:1165-70.

5. Hare HF, Cerny MJ Jr. Soft tissue sarcoma: A review of 200 cases. Cancer 1963;16:1332-7.

6. Linscheid RL, Soule EH, Henderson ED. Pleomorphic Rhabdomyosarcomata of the extremities and limb girdles: A clinicopathological study. J Bone Joint Surg Am 1965;47:715-26.

7. Xarchas K, Papavassiliou N, Tsoutseos N, Burke FD. Rhabdomyosarcoma of the hand: Two case reports and a review of the literature. J Hand Surg Br 1996;21:325-9.

8. Cohen M, Ghosh L, Schafer ME. Congenital embryonal rhabdomyosarcoma of the hand and Apert's syndrome. J Hand Surg Am 1987;12:614-7.

9. SiegelHJ, Connor GS, Lee D, Lopez-BenR, Kelly DR. Synchronous bifocal alveolar rhabdomyosarcoma: A case report. J Bone Joint Surg Br 2006;88:955-8.

10. Sorenson PH, Lynch JC, Qualman SJ, Tirabosco R, Lim JF, Maurer HM, et al. PAX3-FKHR and PAX7-FKHR gene fusions are prognostic indicators in alveolar rhabdomyosarcoma: A report from the children's oncology group. J Clin Oncol 2002;20:2672-9.

11. Fleegler EJ. An approach to soft tissue sarcomas of the hand and upper limb. J Hand Surg Br 1994;19:411-9.

12. Pappo AS, Shapiro DN, Crist WM. Rhabdomyosarcoma. Biology and treatment. Pediatr Clin North Am 1997;44:953-72.

Source of Support: Nil, Conflict of Interest: None declared. 